\title{
Beam steering in superconducting quarter-wave resonators: An analytical approach
}

\author{
Alberto Facco \\ INFN-Laboratori Nazionali di Legnaro, Viale dell'Università 2, I-35020 Legnaro, Padova, Italy, \\ and FRIB, Michigan State University, East Lansing, Michigan 48824, USA \\ Vladimir Zvyagintsev \\ TRIUMF, Vancouver, Canada \\ (Received 11 February 2011; published 26 July 2011)
}

\begin{abstract}
Beam steering in superconducting quarter-wave resonators (QWRs), which is mainly caused by magnetic fields, has been pointed out in 2001 in an early work [A. Facco and V. Zviagintsev, in Proceedings of the Particle Accelerator Conference, Chicago, IL, 2001 (IEEE, New York, 2001), p. 1095], where an analytical formula describing it was proposed and the influence of cavity geometry was discussed. Since then, the importance of this effect was recognized and effective correction techniques have been found [P. N. Ostroumov and K. W. Shepard, Phys. Rev. ST Accel. Beams 4, 110101 (2001)]. This phenomenon was further studied in the following years, mainly with numerical methods. In this paper we intend to go back to the original approach and, using well established approximations, derive a simple analytical expression for QWR steering which includes correction methods and reproduces the data starting from a few calculable geometrical constants which characterize every cavity. This expression, of the type of the Panofski equation, can be a useful tool in the design of superconducting quarter-wave resonators and in the definition of their limits of application with different beams.
\end{abstract}

DOI: 10.1103/PhysRevSTAB.14.070101

PACS numbers: 29.20.Ej, 29.27.- a

\section{INTRODUCTION}

Quarter-wave resonators (QWRs), used to accelerate ion beams with approximately $0.001<\beta<0.2$, are cylindrical and coaxial cavities working in TEM mode which have an up-down asymmetry with respect to the beam axis. To obtain a symmetric accelerating field it is necessary to provide them with axisymmetric beam ports and short accelerating gaps. Along the beam axis, in addition to the usual accelerating field, QWRs have always additional transverse field components, in the vertical direction $y$ parallel to the main resonator axis (electric, $E_{y}$ ) and in the horizontal one $x$ (magnetic, $B_{x}$ ), which both deflect the beam in the vertical direction $y$. This steering effect strongly depends on synchronous phase, beam velocity, accelerating gradient, and resonator geometry [1-6]. This is usually not critical in normal conducting resonators; in superconducting ones, due to the very high gradient and to special constraints in the cavity design, the magnetic component can be very strong and steering could become intolerable, especially for acceleration of high $q / A$ beams [7]. We want to derive a formula, valid for any realistic $E_{a}$, $\phi$, and $\beta$, which describes QWR steering as a function of

Published by the American Physical Society under the terms of the Creative Commons Attribution 3.0 License. Further distribution of this work must maintain attribution to the author(s) and the published article's title, journal citation, and DOI. calculable geometrical constants and transit time factors in the form of simple analytical expressions. This formula should put in evidence the important parameters of this undesirable effect and the suitable correction methods. We will show that, under reasonable approximations, the formula is an extension of the one that was proposed, without derivation, in Ref. [1]. In this paper (differently from Ref. [1]), we will use the standard beam dynamics reference frame, applied to the usual vertical orientation of QWRs with the beam axis located at the lower side of the cavity, and with the origin located on the drift tube axis at the cavity middle.

\section{ANALYTICAL DERIVATION OF STEERING IN QWRS}

To derive an expression for the QWR steering, we will take advantage of the fact that, for symmetry reasons, only three field components are significant along the drift tube axis and in its proximity (see Fig. 1): (1) the accelerating field $E_{z}(z, t)=E_{z}(z) \cos (\omega t)$, with $E_{z}(z)$ antisymmetric with respect to the cavity center; (2) the transverse electric field $E_{y}(z, t)=E_{y}(z) \cos (\omega t)$, with $E_{y}(z)$ symmetric; this component is created by the up-down QWR asymmetry; (3) the transverse magnetic field $B_{x}(z, t)=B_{x}(z) \sin (\omega t)$, with $B_{x}(z)$ antisymmetric; this is the unavoidable magnetic component of the fundamental TEM mode which is used for acceleration in QWRs, which are essentially coaxial transmission lines. 


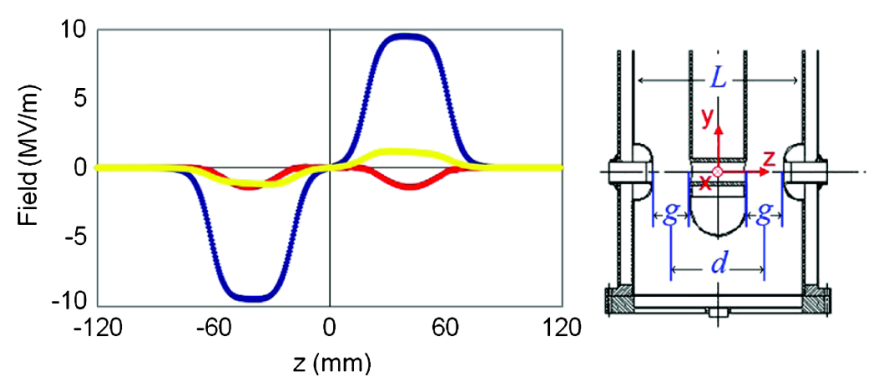

FIG. 1. Left: Typical shape of the field distributions along the beam axis in QWRs. Blue: $E_{z}(z)$; red: $E_{y}(z) \times 10$; yellow: $c B_{x}(z)$. Right: Sketch of the beam port section of a QWR, with the reference frame used in this paper. $L$ is the cavity active length; $g$ and $d$ are the physical gap length and gap-to-gap distance.

The strength of the transverse components usually increases with the cavity aspect ratio (horizontal/vertical dimensions). An example is shown in Fig. 2, where the strengths of the transverse field components normalized to the accelerating one (as defined below) are plotted as a function of their optimum velocity $\beta_{o}$, for $12 \mathrm{QWRs}$ of the type used at INFN-LNL and TRIUMF. These modular cavities, designed for heavy ions acceleration, have all the same active length and similar geometry, and different values of $\beta_{o}$ were obtained by changing their frequency (see Fig. 3). Some of them, up to $\beta_{o}=0.13$, are in operation.

Since the QWR vertical length is $\sim \lambda / 4$ and the cavity diameter is typically $\sim \beta_{0} \lambda$, the QWR aspect ratio is typically $\sim 4 \beta_{0}$. Thus, $B_{x}(z)$ and $E_{y}(z)$ tend to increase linearly with $\beta_{0}$. We can also see that the transverse magnetic force is always 1 order of magnitude larger than the electric one.

The beam deflection $\Delta y^{\prime}$ can be expressed as the ratio $\Delta p_{y} / p_{z}$, where $\Delta p_{y}$ is the transverse momentum kick given by the cavity and $p_{z}$ is the longitudinal momentum of the beam particle.

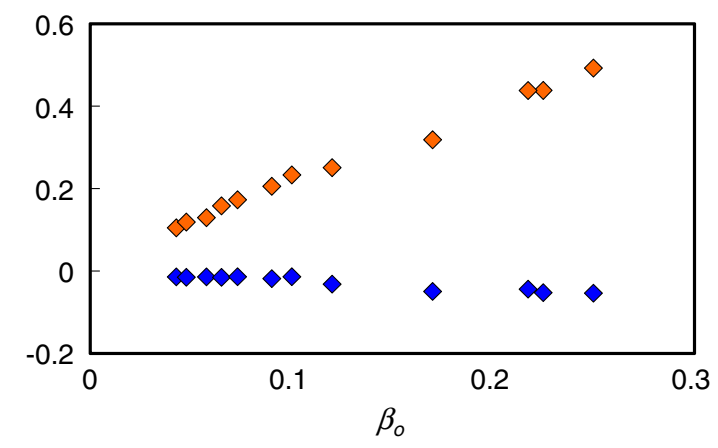

FIG. 2. Relative strength (on axis) of the average transverse electric and magnetic field components in a gap, normalized to the accelerating one (see definition in the text), $E_{y o} / E_{z o}$ (blue) and $c B_{x o} / E_{z o}$ (red), as a function of the QWR optimum $\beta$, calculated for 12 coaxial QWRs of the INFN-TRIUMF type.

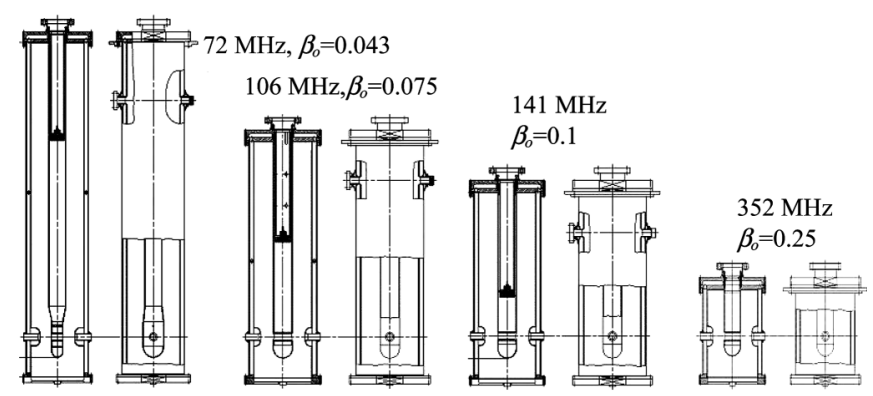

FIG. 3. Examples of INFN-TRIUMF type QWR design for cavities with different frequency and $\beta_{o}$. Cavities with similar geometry, from $\beta_{o}=0.047$ to $\beta_{o}=0.13$, are in use in several superconducting heavy ion linacs.

The deflecting force of the horizontal magnetic field component $B_{x}(z, t)$, perpendicular to the particle velocity, is vertical. Its momentum kick is

$$
\Delta p_{y}=q e \beta c \int_{t(-L / 2)}^{t(L / 2)} B_{x}(z, t) d t,
$$

where $q$ is the charge state of the ion, $e$ is the electron charge, $L$ is the active length of the cavity along the beam axis, and $z$ the particle position at the time $t$. To calculate (1), we can use the classical approximations of a constant beam velocity $\beta$ and of a rectangular field distribution along the beam axis (see, e.g., [8]) which give a simple, but rather precise in the $\beta$ range of interest, analytical solution.

In this case the field inside each gap has a constant amplitude $E_{z}(z)=E_{z o}, \quad E_{y}(z)=E_{y o}, \quad B_{x}(z)=B_{x o}$ for $z>0$, and $E_{z}(z)=-E_{z o}, E_{y}(z)=E_{y o}, B_{x}(z)=-B_{x o}$ for $z<0$. Outside the gaps, $E_{z}(z)=E_{y}(z)=B_{x}(z)=0$. In our standard reference frame we have $E_{z o}>0$ and $B_{x o}>0$, while the sign of $E_{y o}$ could be either positive or negative, depending on the cavity geometry. Assuming that the particle velocity $\beta$ can be considered constant, we can substitute $z=z_{o}+\beta c t$ in the integral and find

$$
\Delta y_{B x}^{\prime}=\frac{2 q e g_{B x} c B_{x o} \sin \phi}{\beta \gamma A m_{o} c^{2}} \sin \left(\frac{\pi d_{B x}}{\beta \lambda}\right) T_{g}\left(\beta, g_{B x}\right),
$$

where

$$
T_{g}\left(\beta, g_{B x}\right) \equiv \frac{\sin \left(\frac{\pi g_{B x}}{\beta \lambda}\right)}{\left(\frac{\pi g_{B x}}{\beta \lambda}\right)}
$$

is the single gap transit time factor [8].

Here $g_{B x}$ is the effective gap length of the $B_{x}(z)$ distribution, $d_{B x}$ is the gap-to-gap distance (center to center), and $\phi=-\omega z_{o} / \beta c$ is the synchronous particle phase. This approximation is usually rather effective and, for real cavities, it is always possible to reproduce the measurements data by properly choosing $B_{x o}$ and "effective" values of $d_{B x}$ and $g_{B x}$, which might be slightly different from the physical ones (called $d$ and $g$ in this paper, see Fig. 1). 
In the case of the transverse electric field component $E_{y}(z, t)$, we have

$$
\Delta p_{y}=q e \int_{t(-L / 2)}^{t(L / 2)} E_{y}(z, t) d t
$$

Again, dividing the result by the particle momentum $p_{z}=\beta \gamma A m_{o} c$ we find the vertical electric deflection:

$$
\Delta y_{E y}^{\prime}=\frac{2 q e g_{E y} E_{y o} \sin \phi}{\beta^{2} \gamma A m_{o} c^{2}} \cos \left(\frac{\pi d_{E y}}{\beta \lambda}\right) T_{g}\left(\beta, g_{E y}\right),
$$

where $g_{E y}$ and $d_{E y}$ are defined as before, but referred to the $E_{y}(z)$ distribution.

It should be noted that magnetic and electric deflection, due to the opposite symmetry of the field distributions which are causing them, are proportional to $\sin (\pi d / \beta \lambda)$ and $\cos (\pi d / \beta \lambda)$, respectively.

Until now we have assumed that all field distributions do not change their sign inside the gap and can be approximated by just a square function. In real QWRs this is true for $E_{z}(z)$ and $B_{x}(z)$, which are related to the normal TEM modes of the resonators, but not always for $E_{y}(z)$ which distribution depends on the cavity geometry in the beam port region. $E_{y}(z)$ may contain two peaks (or even more) with opposite sign inside the gap, and sometimes these peaks are introduced artificially by cavity designers with the aim of canceling the total steering (see below in Fig. 10, top right). Each one of these peaks gives to the total steering a contribution $\Delta y_{E y j}^{\prime}$ that can be expressed with Eq. (5); thus the total electric steering can be better represented by the sum of $n$ contributions $\sum_{J=1}^{n} \Delta y_{E y j}^{\prime}$, in most practical cases with $n=1$ or $n=2$.

The constant $\beta$ and the rectangular field approximations are commonly used also to calculate the ion beam energy gain per nucleon in a cavity (see, e.g., [8]), in the well-known formula (a variant of the Panofsky equation) valid for two-gap cavities working in $\pi$ mode:

$$
\Delta U\left(E_{a}, \phi, \beta\right)=\frac{q e}{A} E_{a} L T(\beta) \cos \phi .
$$

The above approximations allow expressing the transit time factors in an analytical form:

$$
\begin{gathered}
E_{a}=\frac{2 g_{E z} E_{z o}}{L} T_{E z}\left(\beta_{o}\right) \\
T_{E z}(\beta)=\sin \left(\frac{\pi d_{E z}}{\beta \lambda}\right) \frac{\sin \left(\frac{\pi g_{E z}}{\beta \lambda}\right)}{\left(\frac{\pi g_{E z}}{\beta \lambda}\right)}=\sin \left(\frac{\pi d_{E z}}{\beta \lambda}\right) T_{g}\left(\beta, g_{E z}\right) \\
T(\beta)=\frac{T_{E z}(\beta)}{T_{E z}\left(\beta_{0}\right)},
\end{gathered}
$$

where $g_{E z}, d_{E z}$ are the usual effective gap length and gapto-gap distance for the accelerating field distribution $E_{z}(z)$,
$E_{a}$ is the average accelerating field, $T(\beta)$ is the normalized transit time factor, and $\beta_{o}$ the cavity optimum velocity. It is easy to see that the quantities $E_{z o} g_{E z}, c B_{x o} g_{B x}$, and $E_{y o j} g_{E y j}$ have the dimension of a voltage and they are all proportional to the momentum kick given to the particle by the field distributions $E_{z}(z), B_{x}(z)$, and $E_{y j}(z)$, respectively. Thus, defining the new dimensionless geometrical constants

$$
G_{B x} \equiv \frac{c B_{x o} g_{B x}}{E_{z o} g_{E z}} \quad G_{E y j} \equiv \frac{E_{y o j} g_{E y j}}{E_{z o} g_{E z}},
$$

we can express all the transverse kicks as functions of the accelerating voltage in the gap, $E_{z o} g_{E z}$. Using (7) and (10) we can combine (2) and (5) and find an expression which describes beam steering in QWRs as a function of $E_{a}, \phi$, $\beta$, where all the time dependence of the different fields is concentrated in the transit time factors:

$$
\begin{aligned}
\Delta y^{\prime}\left(E_{a}, \phi, \beta\right)= & \frac{q e E_{a} L \sin \phi}{A m_{o} c^{2} T_{E z}\left(\beta_{o}\right)}\left\{\frac{G_{B x}}{\beta \gamma} T_{g}\left(\beta, g_{B x}\right) \sin \left(\frac{\pi d_{B x}}{\beta \lambda}\right)\right. \\
& \left.+\sum_{j=1}^{n} \frac{G_{E y j}}{\beta^{2} \gamma} T_{g}\left(\beta, g_{E y j}\right) \cos \left(\frac{\pi d_{E y j}}{\beta \lambda}\right)\right\} .
\end{aligned}
$$

All geometrical constants in this formula must be calculated along the real particle trajectory.

Steering is maximum in bunching conditions $(\phi=$ $\pm 90 \mathrm{deg}$ ) and disappears at the phase of maximum acceleration $(\phi=0)$. Magnetic steering is proportional to $1 / \beta$ and disappears at $\beta=d_{B x} / \lambda \sim \beta_{o} / 2$, i.e., near half the optimum velocity; electric steering is proportional to $1 / \beta^{2}$ and disappears at $\beta=2 d_{E y} / \lambda \sim \beta_{o}$, near optimum velocity. For a given value of $\phi$, steering is proportional to the energy gain in the resonator. In Fig. 4 the curves calculated with (2), (5), (6), and (11) for a $106 \mathrm{MHz}, \beta=0.075$ QWR are plotted together with data calculated by means of

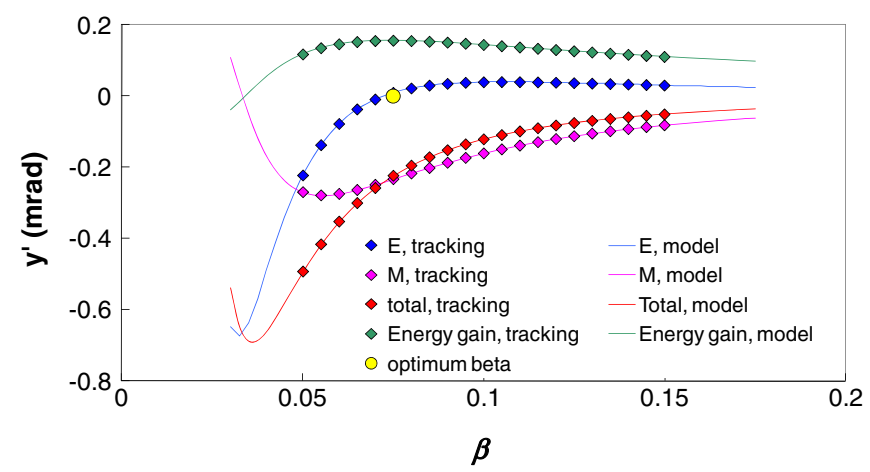

FIG. 4. Energy gain and steering curves $\Delta y^{\prime}(\beta)$ caused by transverse magnetic and electric fields, as well as the total steering, calculated on axis for a $106 \mathrm{MHz}, \beta=0.075 \mathrm{QWR}$ at $1 \mathrm{MV} / \mathrm{m}, q / A=1$, and $\phi=-30^{\circ}$, by means of the analytical model (11) (lines), and numerically by means of particle tracking (diamonds). 
particle tracking in the low field limit (thus at constant $\beta$ ), showing very good agreement.

Although this general expression was based on the assumption of constant $\beta$ in the resonator, it gives a very good approximation whenever the relative change of velocity inside the resonator is small, i.e., if $\Delta \beta / \beta \ll 1$ (see Figs. 4-6), which applies in most practical cases except for very low- $\beta$ cavities working at high gradient with a high $q / A$ beam. When comparing the results of (11) with the steering data calculated with 3D simulation codes, or with the ones obtained from experiments, one should take into account the change of beam velocity in the cavity by assigning to each data point an average value of $\beta$. For $\Delta \beta / \beta \ll 1$, good agreement can be obtained, for example, by defining $\beta \equiv \frac{1}{2}\left(\beta_{\text {in }}+\beta_{\text {out }}\right)$ (see Fig. 5, diamonds).

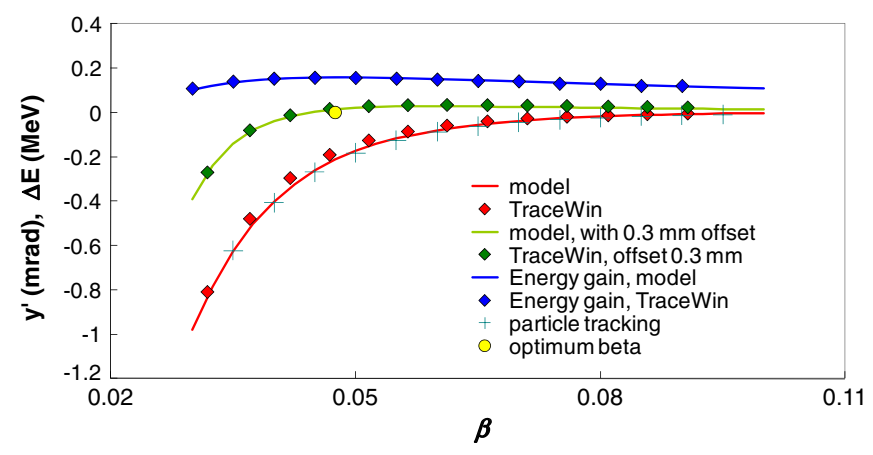

FIG. 5. Proton beam steering and energy gain at $1 \mathrm{MV} / \mathrm{m}$, $\phi=-30^{\circ}$ in a $\beta_{o}=0.047,80 \mathrm{MHz}$ QWR calculated with analytical model (15) (lines), with particle tracking at constant $\beta$ (crosses), and with the 3D beam dynamics code TRACEWIN [11] (diamonds), on axis (red) and with $0.3 \mathrm{~mm}$ beam offset (green) which provides steering compensation. The $\beta$ values used for the TRACEWIN data are the average particle velocities in the resonator.

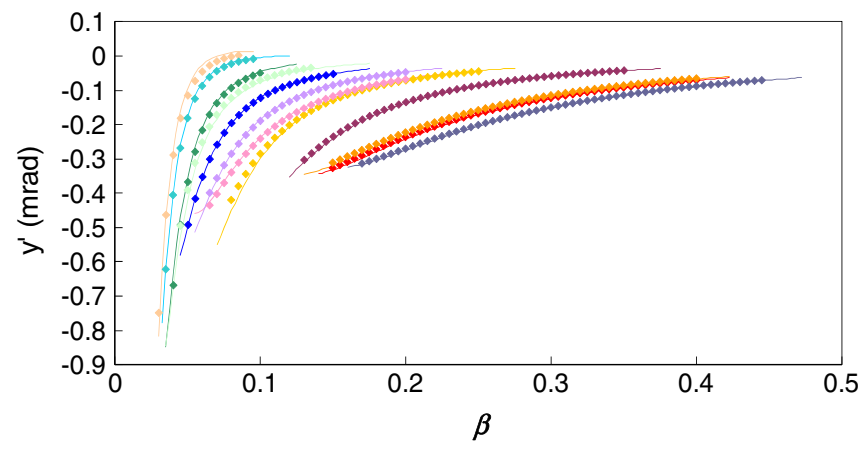

FIG. 6. On-axis steering vs $\beta$ in the 12 INFN-TRIUMF type QWRs of Fig. 2 with $\beta_{o}$ from 0.043 to 0.25 , at $1 \mathrm{MV} / \mathrm{m}$, $A / q=1$, and $\phi=-30^{\circ}$, calculated with particle tracking (diamonds) and by means of expression (11) (lines), in the range from $0.75 \beta_{o}$ to $2 \beta_{o}$.
When $n=1$ (i.e. when $E_{y}$ consists of only one peak per gap), if the effective gap lengths and gap-to-gap distances are approximated with the purely physical ones $d$ and $g$, this expression becomes particularly simple:

$$
\Delta y^{\prime}(\beta)=\frac{q e E_{a} L T(\beta) \sin \phi}{A m_{0} c^{2}}\left\{\frac{G_{B x}}{\beta \gamma}+\frac{G_{E y}}{\beta^{2} \gamma \operatorname{Tan}\left(\frac{\pi d}{\beta \lambda}\right)}\right\} .
$$

Taking into account the rotated reference frame and reintroducing the effective distance $d_{E y},(12)$ is equivalent to the formula of Ref. [1].

A mechanism which is always present in a cavity is rf defocusing, which deflects the beam by an amount which is proportional to the distance $r$ of the beam from its canonical axis (see e.g. [9]). In Eq. (11) rf defocusing is already included if the parameters are calculated along the real beam trajectory; in this case, also when the beam has a vertical offset $y \neq 0$ the steering is reproduced correctly. However, it is often desirable to characterize the cavity only with one set of parameters calculated along its geometrical axis, where $\mathrm{rf}$ defocusing is absent. This can be obtained by adding to (11) a term describing rf defocusing:

$$
\Delta y_{\mathrm{rf}}^{\prime}(\beta)=-\frac{\omega}{2 \beta^{3} \gamma^{3} m_{0} c^{3}} \frac{q}{A} e E_{a} L T(\beta) y \sin \phi .
$$

This is a good approximation if $y \ll R$, where $R$ is the beam port aperture radius.

Since $\Delta y_{\mathrm{rf}}^{\prime}(\beta)$ is proportional to $E_{a} L T(\beta) \sin \phi$ and the longitudinal transit time factor depends on the effective lengths $g_{E z}, d_{E z}$ of the accelerating field distribution, the term (13) can be included in (11) in a very natural way.

Defining

$$
\begin{aligned}
D_{B x}(\beta) & =\frac{G_{B x}}{\beta \gamma} T_{g}\left(\beta, g_{B x}\right) \sin \left(\frac{\pi d_{B x}}{\beta \lambda}\right) \\
D_{E y j}(\beta) & =\frac{G_{E y j}}{\beta^{2} \gamma} T_{g}\left(\beta, g_{E y j}\right) \cos \left(\frac{\pi d_{E y j}}{\beta \lambda}\right) \\
D_{\mathrm{rf}}(\beta, y) & =-\frac{\omega}{2 \beta^{3} \gamma^{3} c} T_{g}\left(\beta, g_{E z}\right) \sin \left(\frac{\pi d_{E z}}{\beta \lambda}\right) y
\end{aligned}
$$

and using (7)-(9), we find a formula of the desired form which can represent, when $y \ll R$, the beam steering in QWRs, with all constants calculated along the resonator geometrical axis:

$$
\begin{aligned}
\Delta y^{\prime}\left(E_{a}, \phi, \beta, y\right)= & \frac{q e E_{a} L \sin \phi}{A m_{o} c^{2} T_{E z}\left(\beta_{0}\right)} \\
& \times\left\{D_{B x}(\beta)+\sum_{J=1}^{n} D_{E y j}(\beta)+D_{\mathrm{rf}}(\beta, y)\right\} .
\end{aligned}
$$




\section{CALCULATION OF THE GEOMETRICAL CONSTANTS}

The main advantage of (15) is that steering is completely defined by a few geometrical constants which are related to different field components, and can be eliminated by optimizing one or two of them. In order to do it with real cavities, it is necessary to have a consistent and reliable way to extract the necessary parameters from the field distributions $E_{z}(z), B_{x}(z), E_{y j}(z)$ calculated along the beam port axis by means of a simulation code. One way is making a best fit of all parameters in order to reproduce $\Delta y^{\prime}(\beta)$ obtained with a 3D beam dynamics simulation code with realistic fields. However, good results can be obtained by a simple weighted mean of the field distributions data. The following formulas can be used (for sake of simplicity, when possible, all indexes are represented by $\alpha$ and all fields $E_{z}(z), B_{x}(z)$, $E_{y j}(z)$ by $\left.F_{\alpha}(z)\right)$.

(a) Geometrical constants $G_{B x}, G_{E y j}$ :

$$
G_{B x}=\frac{c \int_{0}^{L / 2} B_{x}(z) d z}{\int_{0}^{L / 2} E_{z}(z) d z} \quad G_{E y j}=\frac{\int_{0}^{L / 2} E_{y j}(z) d z}{\int_{0}^{L / 2} E_{z}(z) d z} .
$$

(b) Gap-to-gap distance $d_{E z}, d_{E y j}, d_{B x}$ :

$$
d_{\alpha}=2 \frac{\int_{0}^{L / 2} z F_{\alpha}(z) d z}{\int_{0}^{L / 2} F_{\alpha}(z) d z} .
$$

(c) Gap length $g_{E z}, g_{E y j}, g_{B x}$ :

$$
g_{\alpha}=4 \frac{\int_{0}^{L / 2}\left|z-\frac{d_{\alpha}}{2}\right| F_{\alpha}(z) d z}{\int_{0}^{L / 2} F_{\alpha}(z) d z} .
$$

Once defined the gap length, the field amplitudes $E_{z o}, E_{y j o}$, $B_{x o}$ can be calculated:

$$
F_{\alpha o}=\frac{1}{g_{\alpha}} \int_{0}^{L / 2} F_{\alpha}(z) d z
$$

Starting from the cavity simulation data, $E_{z o}$ and $E_{y j o}$ must be calculated at the phase in which $E_{z}(z)$ is maximum, in order to preserve the right sign of $E_{y j o}$, while $B_{x o}$ must be calculated with $90^{\circ}$ delay and must be positive. In the case of the transverse electric field $E_{y}(z)$, when it consists of more than one peak (e.g. due to the beam port tilting, see below), every single peak should be used independently (nulling all points out of the peak) to calculate $G_{E y j}, d_{E y j}$, and $g_{E y j}$ in order to be consistent with the used approximation. This is not difficult, however, since the contributions of the wall tilting are very well defined in space [2].

Definitions (17) and (18) have been chosen in order to obtain the exact values of gap length $g_{\alpha}$ in the case of ideal square field distribution [thus, in case of a Gaussian-like field distribution $F_{\alpha}(z)=F_{\alpha o} e^{-\left[(z-d / 2)^{2} / 2 \sigma^{2}\right]} /(\sigma \sqrt{2 \pi})$, the gap length would become $g_{\alpha} \sim 4 \sigma$ ]. This choice, dictated by analytical simplicity, could appear somehow arbitrary but it works rather well in real cases because expressions (11) and (15) are not very sensitive to the shape of the single gap distributions (provided that it consists of a single peak), except for $\beta \ll \beta_{o}$, thus out of the usable range of the QWR.

In Figs. 5 and 6 the steering curves $\Delta y^{\prime}(\beta)$, at $q / A=1$, $E_{a}=1 \mathrm{MV} / \mathrm{m}$, and $\phi=-30^{\circ}$, are shown for a set of cavities with $0.043 \leq \beta_{o} \leq 0.25$ and frequency from 72 to $352 \mathrm{MHz}$. The curves, calculated with (15), show good agreement with the particle tracking data (diamonds) and also with data calculated with a 3D beam dynamics code (Fig. 5). All geometrical constants have been calculated as described in this paragraph. Around optimum $\beta$, if no correction is applied, steering is around $0.2 \div 0.3 \mathrm{mrad} /[(q / A)(\mathrm{MV} / \mathrm{m})]$ for all cavities of this particular type, and increases fast at decreasing of $\beta$.

\section{DISCUSSION OF THE CORRECTION METHODS}

The analytical model which was previously described allows a simple discussion and numerical evaluation of the correction methods applied in QWRs.

\section{A. Axisymmetric drift tube}

The addition of a "donut-shaped" axisymmetric drift tube at the inner conductor of a coaxial cavity has the main effect of reducing the magnetic field in the gap (see Fig. 7), and consequently the total steering [1,3]. In expression (15) this is shown by the reduction of the geometrical constant $G_{B x}$ value from 0.25 to 0.16 in the cavity of Fig. 7.

\section{B. Beam offset}

By means of a proper vertical offset of the beam, rf defocusing (13) can be used to counteract the transverse electric and magnetic steering [2]. The net effect of all

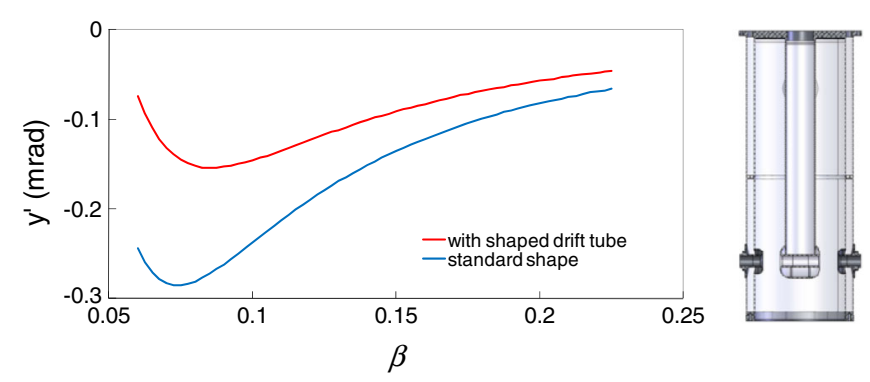

FIG. 7. Left: Magnetic steering in a $\beta=0.11,141 \mathrm{MHz}$ coaxial QWR at $1 \mathrm{MV} / \mathrm{m}, A / q=1$, and $\phi=-30^{\circ}$. Blue line: Normal cavity with $G_{B x}=0.25$; red line: the same cavity after the addition of an axisymmetric "donut" shaped drift tube, leading to $G_{B x}=0.16$. Right: Sketch of the resonator with the modified drift tube. 


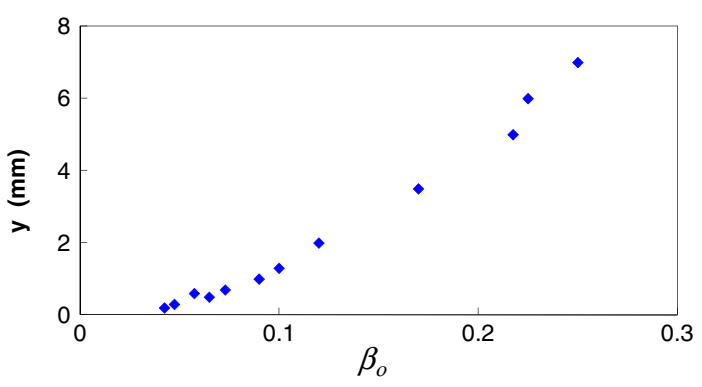

FIG. 8. Optimum beam offset $y$ vs $\beta_{o}$ for steering reduction below $0.1 \mathrm{mrad} /[(\mathrm{MV} / \mathrm{m})(q / A)]$ in the set of 12 QWRs of Fig. 6, calculated with formula (15). At higher $\beta_{o}$ the required offset approaches the beam port aperture radius.

steering components results just in the displacement of the optimum electromagnetic axis of the cavity (one of the reasons why QWRs steering was not observed for a long time in heavy ion linacs is probably that it was mistaken for rf steering caused by misalignment).

For a given offset $y$, the reduction factor varies with $\beta$ but it is the same for any cavity gradient or synchronous phase. This correction method is very effective in a rather wide range of $\beta$ (see Figs. 5 and 9, green lines, and Fig. 10, light blue line) and can be applied with any QWR. Because of its inverse dependence on $\beta^{3}$, however, the required offset is moderate only in low- $\beta$ cavities, while in high- $\beta$ ones it could become comparable to the beam port aperture, i.e. $y \sim R$ (see Fig. 8). This would be hardly acceptable due to reduction of the effective aperture and to possible field distortion. It should be noted that, in this case, the beam port edge effect becomes significant and the rf defocusing term in (15) is not anymore a good approximation, while formula (11) still gives an accurate description of the steering if the parameters (16)-(18) are calculated along the real beam trajectory (Fig. 9).

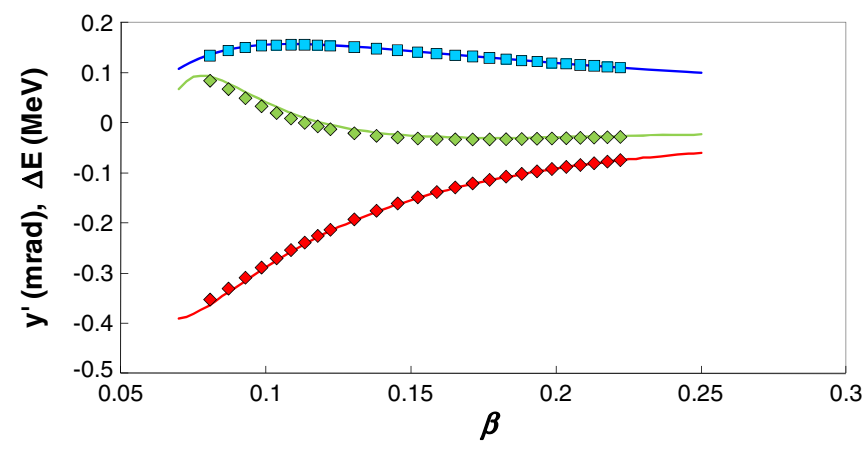

FIG. 9. Energy gain and steering in a $160 \mathrm{MHz}, \beta_{o}=0.11$ QWR. Red line: analytical model, beam on axis; green line: analytical model, with the parameters calculated with expression (11) along the real beam axis after cavity vertical displacement of $1.7 \mathrm{~mm}$ for steering correction. Diamonds: Results obtained by the code TRACEWIN and realistic 3D field maps.
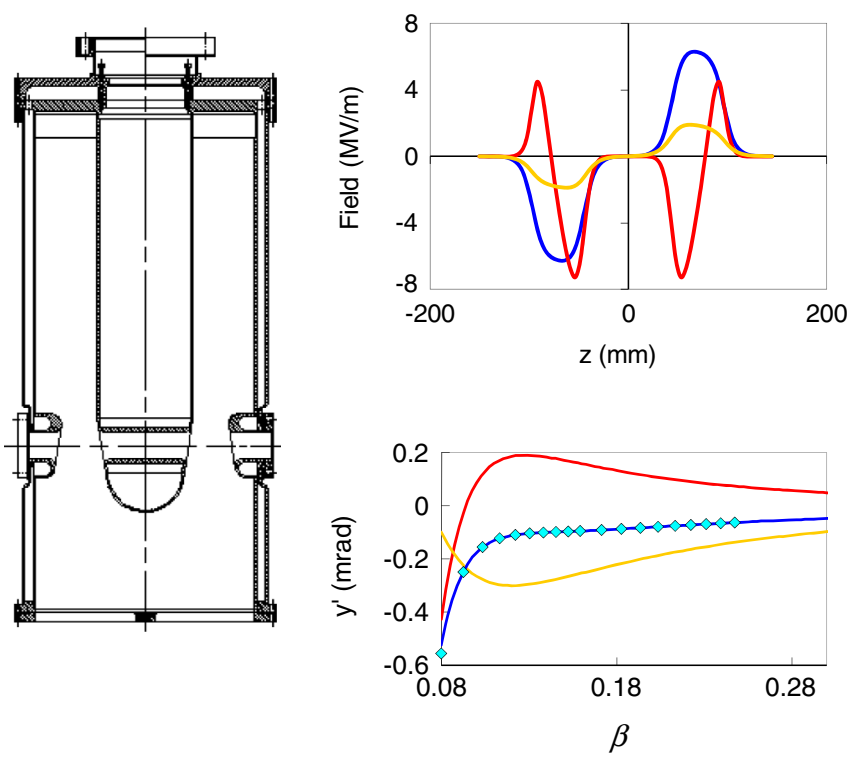

FIG. 10. Example of $\beta=0.16,161 \mathrm{MHz}$ prototype cavity with tilted beam ports for steering correction [12]. Right top: field distributions $E_{z}$ (blue), $c B_{x}$ (yellow), and $E_{y} \times 10$ (red line) with the correcting bumps generated by 9 degrees beam port tilting. Right bottom: steering at $1 \mathrm{MV} / \mathrm{m}, \phi=-30^{\circ}$ for protons in the QWR with beam port tilting. Blue diamonds: total steering calculated by TRACEWIN and realistic 3D field maps. The lines represent the model results: electric (red), magnetic (yellow), and total steering (blue), fitting rather well the 3D simulation results.

\section{Beam port tilting}

The geometrical constants $G_{E y j}$ and $G_{B x}$, which determine the steering strength, can be reduced to some extent by modifying the cavity shape [1,3], but usually the magnetic component cannot be eliminated completely. A smart correction method developed at ANL [2], which can be applied also in high- $\beta$ QWRs [10], is based on the creation of artificial $E_{y}$ components able to counteract the steering; this can be done by properly shaping the cavity walls at the beam ports positions, both at the outer and inner conductors. By tilting the surface with respect to the plane perpendicular to the beam axis it is possible to create new $E_{y}(z)$ components, of either positive or negative sign (see an example in Fig. 10, right top, red curve).

Each one of the two symmetric pairs of tilted beam ports (i.e. the pair on the inner conductor and the pair on the outer conductor) gives an extra kick $\Delta y_{E y j}^{\prime}(\beta)$ represented in (15) by the two parameters $G_{E y 1}$ and $G_{E y 2}$ (produced by the inner and outer conductor tilted beam ports, respectively). Optimizing them it is usually possible to obtain very good cancellation in the range which is useful for acceleration (approximately $0.7 \beta_{o}<\beta \leq 2 \beta_{o}$ ), while steering becomes rapidly intolerable while moving below $\beta_{o}$ (Fig. 11, yellow line). 


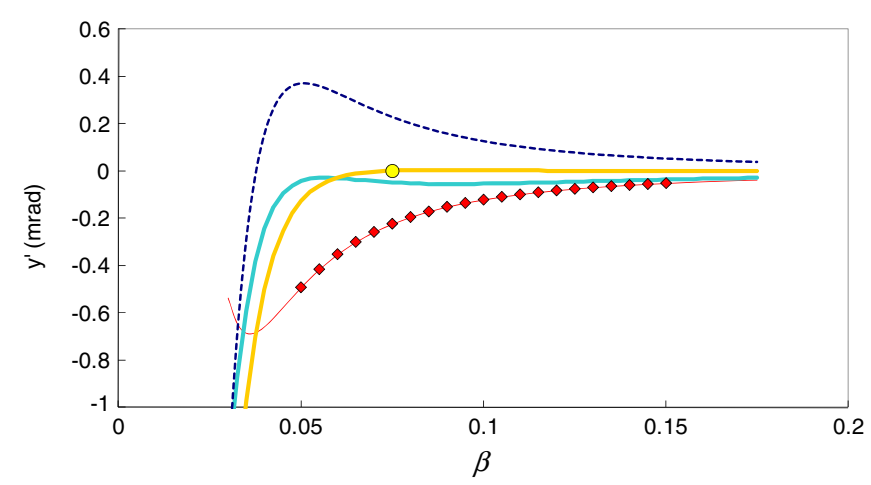

FIG. 11. Steering in a $106 \mathrm{MHz}, \beta=0.075$ at $1 \mathrm{MV} / \mathrm{m}$, $q / A=1$ and $\phi=-30^{\circ}$, calculated with expression (15). Red line: model without correction; red diamonds: particle tracking without correction; blue line: corrected with $0.7 \mathrm{~mm}$ axis displacement; yellow line: corrected by optimizing the $G_{E y 2} G_{E y 3}$ parameters. The dashed line represents the contribution of the correcting field component generated by the tilted beam ports.

This method is applicable when $\left|E_{y j o}\right| \ll E_{z o}$, otherwise the correcting field would be comparable to the accelerating one, requiring a very large tilting angle and consequently loss of acceleration efficiency and field distorsion. In the example of Fig. 10, a complete cancellation of the steering would have required a significant increase of the inner conductor tilting angle.

The optimum values of the two correcting fields $E_{y 1 o}$ and $E_{y 2 o}$ (normalized to the longitudinal one $E_{z o}$ ), created by the beam port tilting for steering compensation and calculated with (15) for the usual 12 coaxial cavities, are shown in Fig. 12.

In order to compare the data in cavities with different $\beta$, but with similar geometrical characteristics, we have approximated the effective gap lengths of the correcting field $g_{E y 1}, g_{E y 2}$ with the same value of $20 \mathrm{~mm}$ (half the gap length). The best cancellation was usually obtained with

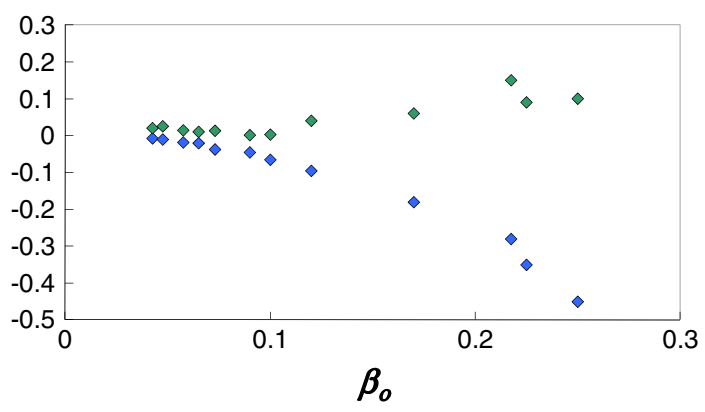

FIG. 12. Average electric fields (normalized to the longitudinal one) vs $\beta$ of the peaks required for steering correction in 12 QWRs, calculated by means of expression (15). Blue diamonds: $E_{y 1 o} / E_{z o}$ generated by the tilted drift tube edge; green diamonds: $E_{y 2 o} / E_{z o}$ generated by the tilted beam port edge. At the higher values of $\beta$ the required transverse electric field amplitude approaches the one of the accelerating field.
$E_{y 1 o} \neq E_{y 2 o}$. However, the optimum $E_{y 2 o}$ values increase fast with $\beta_{o}$, showing in the $\beta_{o}=0.25$ cavity a required transverse correcting field comparable to the accelerating one. This makes also this correction method hardly applicable in that particular resonator.

Although probably unnecessary in traditional low- $\beta$ heavy ion linacs, the combination of drift tube shaping, beam offset, and beam port tilting allows significant extension of the QWR capabilities to high $\beta$ and low $A / q$ beams. The above calculations based on the analytical model suggest that steering can be efficiently reduced just by beam offset in cylindrical QWRs up to about $\beta_{o} \sim 0.1$, while beam port tilting and drift tube shaping can extend this range to about $\beta_{o} \sim 0.15$. Correction methods are increasingly more difficult to apply at higher $\beta_{o}$.

\section{CONCLUSIONS}

Beam steering in quarter-wave resonators can be calculated with good approximation for any $\beta, \phi$ of the beam and for any cavity gradient $E_{a}$ by means of the relatively simple analytical formula that was derived in this paper. This formula is conceptually equivalent, but related to beam steering in QWRs, to the classical formula of the energy gain in a resonator which includes the analytical expression of the transit time factor, and utilizes the same approximations. Any cavity can be described with a set of constants which can be easily extracted from EM simulation data. Each constant is related to a particular field component, including the ones artificially introduced for steering correction, allowing a straight comprehension of the effect on the beam produced by any change in the cavity geometry. We have discussed the characteristics of the QWR steering and the range of applicability of the correction techniques by means of this analytical model. This tool can be useful for cavity design and for cavity characterization, and in the choice of the cavity type for different applications.

[1] A. Facco and V. Zviagintsev, in Proceedings of the Particle Accelerator Conference, Chicago, IL, 2001 (IEEE, New York, 2001), p. 1095.

[2] P. N. Ostroumov and K. W. Shepard, Phys. Rev. ST Accel. Beams 4, 110101 (2001).

[3] P. N. Ostroumov and K. W. Shepard, in Proceedings of the 21st International Linac Conference, Gyeongju, Korea, 2002 (Pohang Accelerator Laboratory, Pohang, Korea, 2002), p. 473.

[4] D. Gorelov et al., in Proceedings of the 21st International Linac Conference, Gyeongju, Korea, 2002 (Ref. [3]), p. 365.

[5] M. Pasini, R.E. Laxdal, and P.N. Ostroumov, in Proceedings of the 8th European Particle Accelerator Conference, Paris, 2002 (EPS-IGA and CERN, Geneva, 2002), p. 933. 
[6] M. A. Fraser, R. M. Jones, and M. Pasini, Phys. Rev. ST Accel. Beams 14, 020102 (2011).

[7] W. Bräutigam, O. Felden, M. Glende, H. Jungwirth, A. Lehrach, R. Maier, S. Martin, A. Schnase, Y. Senichev, R. Stassen, R. Tölle, E. Zaplatin, A. Facco, and V. Zvyagintsev, in Proceedings of the 10th Workshop on RF Superconductivity, 2001, Tsukuba, Japan, pp. 29-33.

[8] T.P. Wangler, $R f$ Linear Accelerators (Wiley \& Sons Publishers, New York, 1998), pp. 36-46 and 89-90.
[9] Wangler, Rf Linear Accelerators (Ref. [8]), p. 197.

[10] J. D. Fuerst, in Proceedings of SRF2009, Berlin, Germany, p. 52.

[11] R. Duperrier, N. Pichoff, and D. Uriot, ICCS Conference, Amsterdam, 2002.

[12] V. Zviagintsev, A. Facco, C. C. Compton, T. L. Grimm, W. Hartung, F. Marti, and R. York, Proceedings of the 11th Workshop of Rf Superconductivity SRF2003, Travemunde, 2003, p. 297. 